\title{
Propagating gene expression fronts in a one-dimensional coupled system of artificial cells
}

\author{
Alexandra M. Tayar ${ }^{1 \dagger}$, Eyal Karzbrun ${ }^{1 \dagger}$, Vincent Noireaux ${ }^{2}$ and Roy H. Bar-Ziv ${ }^{1 \star}$
}

\begin{abstract}
Living systems employ front propagation and spatiotemporal patterns encoded in biochemical reactions for communication, self-organization and computation ${ }^{1-4}$. Emulating such dynamics in minimal systems is important for understanding physical principles in living cells ${ }^{5-8}$ and in vitro9-14. Here, we report a one-dimensional array of DNA compartments in a silicon chip as a coupled system of artificial cells, offering the means to implement reaction-diffusion dynamics by integrated genetic circuits and chip geometry. Using a bistable circuit we programmed a front of protein synthesis propagating in the array as a cascade of signal amplification and short-range diffusion. The front velocity is maximal at a saddle-node bifurcation from a bistable regime with travelling fronts to a monostable regime that is spatially homogeneous. Near the bifurcation the system exhibits large variability between compartments, providing a possible mechanism for population diversity. This demonstrates that on-chip integrated gene circuits are dynamical systems driving spatiotemporal patterns, cellular variability and symmetry breaking.
\end{abstract}

Sharp travelling fronts are prevalent in nature and emerge when nonlinear and dispersive effects combine, for example solitary waves in nonlinear optics, fluid convection, and chemical reactions ${ }^{15}$. Biological multicellular systems use travelling fronts of molecular signals as a means for computation and communication over long distances when signalling by diffusion is inefficient. In these systems, cells can be described as autocatalytic units that amplify and transmit signals beyond a threshold. Because signals dissipate in the medium over short distances, long-range information transmission is achieved by consecutive local cell-cell interactions. Signal propagation has been measured over orders of magnitudes in a variety of biological processes, ranging from fast action potentials in neuron networks with typical velocities ${ }^{16}$ of $v \approx 10^{7} \mathrm{~mm} \mathrm{~h}^{-1}$, to slow gene expression fronts in development ${ }^{3}$ with $v \approx 0.1 \mathrm{~mm} \mathrm{~h}^{-1}$. Although some systems have a linearly unstable initial state, and thus can be treated as Fisher waves ${ }^{17}$, the majority of biological examples require more complex models, including the cable equation and Hodgkin-Huxley model ${ }^{18}$. These systems raise questions concerning the selected speed, bifurcations, fluctuations and stability in parameter space ${ }^{15}$, which are challenging to study in a living organism.

Front propagation has been extensively studied in chemical reactions ${ }^{16,19}$ and more recently in reconstituted biological systems, including the bacterial cell division network ${ }^{10}$, the Xenopus laevis cell cycle ${ }^{11}$, and RNA (ref. 20) and DNA (ref. 14) catalytic reactions. In the past decade cell-free transcription-translation reactions have been used to advance the design of programmable artificial gene systems, including bulk solution ${ }^{21}$, gels ${ }^{9}$, membrane vesicles $^{22}$, water-in-oil drops ${ }^{23}$, microfluidic devices ${ }^{24}$ and on-chip
DNA compartments ${ }^{13}$. So far, however, a synthetic spatially coupled cellular system, driven by integrated programmable gene expression circuits and capable of long-range communication, has not been reconstructed. Here, we used our recently developed on-chip DNA compartments $^{13}$ to design a one-dimensional array of coupled artificial cells programmed with a bistable genetic circuit to exhibit front propagation.

The array consisted of 15 compartments connected to a main flow channel and interconnected by fork-shaped capillaries. The array was carved in silicon to a shallow depth $h=2-3 \mu \mathrm{m}$, with compartment radius $R=50 \mu \mathrm{m}$, capillary lengths $L_{1}=150 \mu \mathrm{m}$, $L_{2}=400 \mu \mathrm{m}, L_{3}=50 \mu \mathrm{m}$, and width $W=10 \mu \mathrm{m}$. The main flow channel was $50 \mu \mathrm{m}$ deep and $900 \mu \mathrm{m}$ wide (Fig. 1a and Supplementary Fig. 1). Gene circuits were patterned in every compartment, the device was sealed, and Escherichia coli cell extract continuously flowed through the main channel ${ }^{13}$. Protein synthesis initiated by diffusion of cell extract from the flow channel through the capillaries and into the compartments (Methods). Synthesized proteins were evacuated at the capillary opening to the flow channel, setting a boundary condition of zero protein concentration.

The expression-diffusion dynamics were characterized by a separation of timescales between the diffusion of proteins along the capillaries, which reached steady-state profiles within minutes, and the gene expression dynamics, which changed over hours ${ }^{13}$ (Supplementary Information). Thus, we observed steady-state linear concentration gradients along the capillaries and a homogeneous protein concentration, $p_{i}$, within each compartment, $i=1-15$, throughout the expression dynamics (Supplementary Fig. 2). The protein flux out of each compartment can be derived by Fick's law, $J=-D_{0} \nabla p_{i}=-p_{i} / \tau$, setting a geometrically defined protein lifetime, $\tau=\left(\pi R^{2} L_{1} / D_{0} W\right) \Phi_{1}\left(L_{1} / L_{2}, L_{2} / L_{3}\right) \approx 0.4 \mathrm{~h}$, where $D_{0} \approx 0.126 \mathrm{~mm}^{2} \mathrm{~h}^{-1}\left(3.5 \times 10^{-7} \mathrm{~cm}^{2} \mathrm{~s}^{-1}\right)$ is the protein diffusion constant and $\Phi_{1} \approx 0.5$ (Supplementary Equations $1-20$ ). In general, $0<\Phi_{1}<1$ depends on the inter-compartment connections, and for the case of isolated compartments $\Phi_{1}\left(L_{2} / L_{1} \gg 1\right)=1$ (Supplementary Equation 20).

The diffusion flux between compartments is determined by the fork-shaped capillaries, $\left(L_{2}, L_{3}\right)$, and can be estimated by applying Kirchhoff current conservation law at each junction and Fick's law with a linear concentration gradient along the capillaries (Supplementary Equations 8-21). This leads to a discrete one-dimensional diffusion term for $p_{i}$ in the $i$ th compartment, $\Delta p_{i}=D\left(\left(p_{i-1}+p_{i+1}-2 p_{i}\right) / L_{2}^{2}\right)$, with an effective diffusion coefficient, $D=D_{0}\left(L_{2} W / \pi R^{2}\right) \Phi_{2}\left(L_{2} / L_{3}\right) \approx 0.02 \mathrm{~mm}^{2} \mathrm{~h}^{-1}$ and $\Phi_{2}=0.28$ (Supplementary Equation 21). For short shunts, $\Phi_{2}\left(L_{3} \ll L_{2}\right) \rightarrow 0$, diffusion between compartments is negligible compared to diffusion to the main channel, which approached the limit of isolated compartments ${ }^{13}$.

${ }^{1}$ Department of Materials and Interfaces, Weizmann Institute of Science, Rehovot 76100, Israel. ${ }^{2}$ Department of Physics, University of Minnesota, Minneapolis, Minnesota 55455, USA. †These authors contributed equally to this work. *e-mail: roy.bar-ziv@weizmann.ac.il 
a

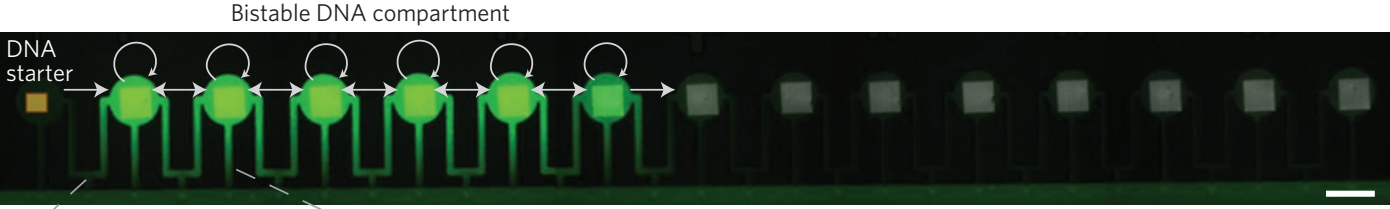

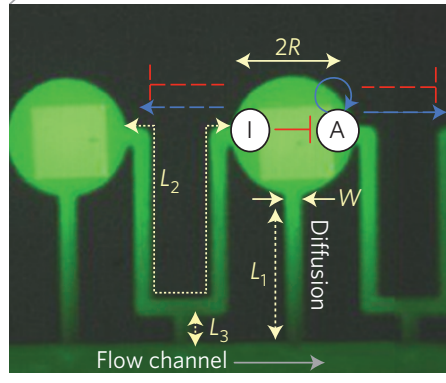

\section{c}

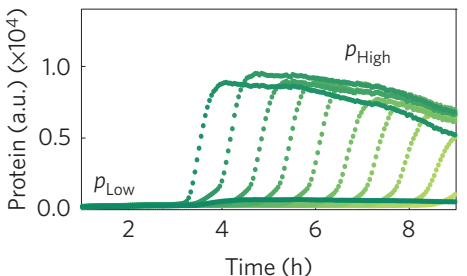

d

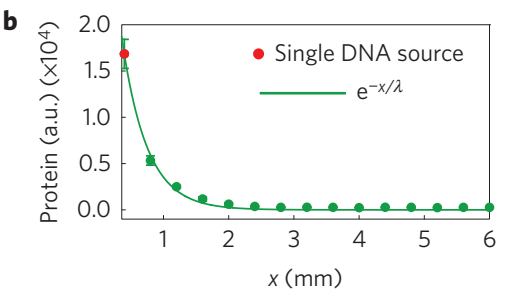

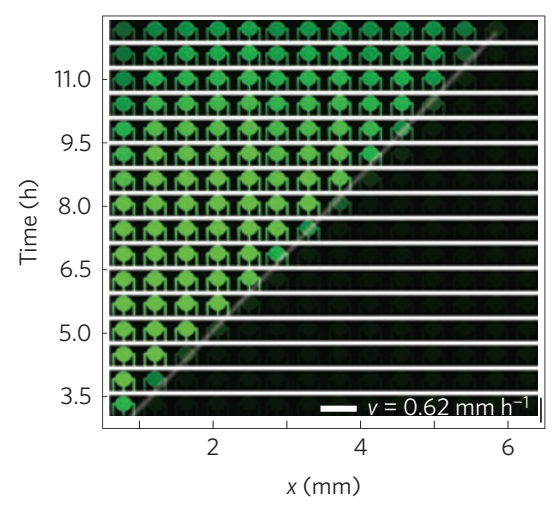

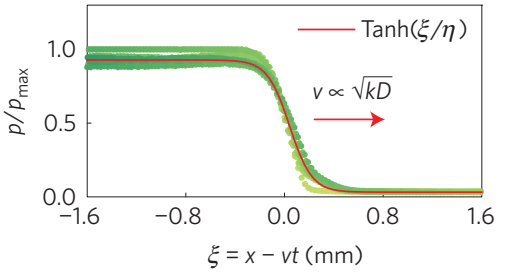

f

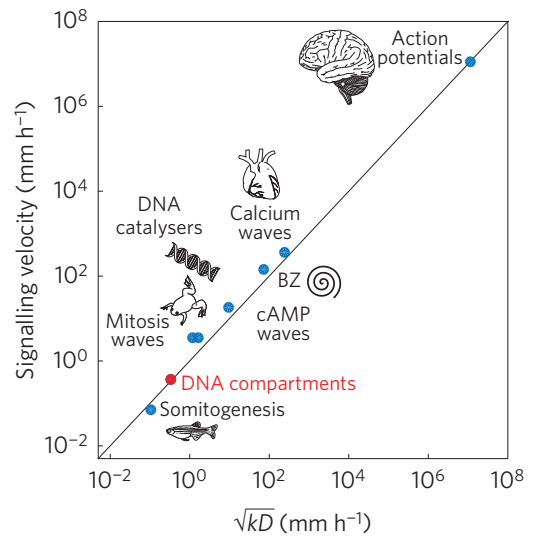

Figure 1 | Travelling gene expression front in an array of coupled DNA compartments. a, Overlay image of expressed GFP and fluorescently labelled DNA patterns (white squares). Compartments 2-15 (from left) were patterned with a bistable gene circuit; the first one patterned with a small amount of starter construct to initiate propagation. Scale bar, $100 \mu \mathrm{m}$. Inset: single compartment overlaid with the self-activator/inhibitor (A/I) scheme using activator $\sigma^{28}$ and inhibitor $A \sigma^{28}$. b. GFP concentration profile in arbitrary units (a.u.) along 15 compartments formed from a single DNA source in the first compartment. Solid line is an exponential fit, $\mathrm{e}^{-x / \lambda}$, with $\lambda=0.416 \pm 0.016 \mathrm{~mm}(1.04 \pm 0.04$ compartments) averaged over $4 \mathrm{~h}$ of expression. c, Expression dynamics of the propagator during $9 \mathrm{~h}$ of experiment. Each graph represents the profile in a compartment according to their order in the array. $\mathbf{d}$, A spacetime image of GFP in the array propagating at a constant velocity of $v=0.62 \mathrm{~mm} \mathrm{~h}^{-1}\left(1.55\right.$ compartment $\left.\mathrm{h}^{-1}\right)$. e, Collapse of fronts in the travelling frame of reference, $\xi=x-v t$. Protein levels are normalized to the maximal protein value in the array for each time point (Supplementary Information). Red line is a fit to $\operatorname{Tanh}(\xi / \eta)$ with $\eta \approx 0.2 \mathrm{~mm}$ ( 0.5 compartment). f, Propagation velocity for a range of biological systems as a function of scaling parameter $\sqrt{k D}$, where $D$ is the diffusion constant of the propagating signal, and $k$ is the typical autocatalytic rate of each system. The DNA compartment array is denoted in red. Exact range appears in Supplementary Table 1.

The diffusion between compartments, effective protein lifetime, and nonlinear interactions dictated by the genetic circuit can all be summarized in a discrete one-dimensional reactiondiffusion equation:

$$
\partial_{t} p_{i}=\Delta p_{i}-\frac{p_{i}}{\tau}+f\left(p_{i}\right)
$$

Here, $f\left(p_{i}\right)$ is the protein synthesis rate in response to the local protein concentration $p_{i}$, and is determined by the genetic circuit. The array was designed to obtain nearest-neighbour interactions, with a diffusion length smaller than a single compartment, $\sqrt{D \tau} \approx 0.1 \mathrm{~mm}<L_{2}$, (Supplementary Equations $22-24$ ). Indeed, we measured an exponentially decaying protein concentration gradient from a single DNA source with a decay length of $\lambda \approx 0.4 \mathrm{~mm}$ (one compartment) along the array (Fig. 1b).

To program signal propagation we designed a bistable gene circuit comprising an activator in a self-feedback loop, and its inhibitor (Fig. 1a, inset, Supplementary Fig. $3{ }^{25}$. The circuit dynamics attained one of two steady-state protein expression levels reported using green fluorescent protein (GFP): high $p_{\text {High }} \approx 0.5 \mu \mathrm{m}$, or $p_{\text {Low }}$ of concentration lower than detection limit $(\approx 50 \mathrm{nM}$; Supplementary Fig. 4). In the absence of the inhibitor a small leak initiates the positive feedback and the circuit dynamics has a single steady-state solution, $p_{\text {High }}$. The circuit is modelled accounting for the competition over gene expression machinery (Supplementary Equations 25-40), resulting in a circuit response function $f\left(p_{i}\right)$ for the activator dynamics, $p_{i}$, (Supplementary Equation 40 ). The bistable circuit was patterned in all compartments except for the anterior one, which was patterned with a 'starter' gene constantly expressing the activator at concentration lower than $p_{\text {High }}$.

During the first $3.5 \mathrm{~h}$ of expression all compartments maintained $p_{\text {Low }}$ (Fig. $1 \mathrm{c}, \mathrm{d} t<3.5 \mathrm{~h}$ ). Signalling was initiated from the anterior compartment, followed by diffusion of activator to the neighbouring compartment. This triggered the autocatalytic reaction, switching the neighbouring compartment to $p_{\text {High }}$ at $t=3.5 \mathrm{~h}$, and creating a new source of activator. The cycle of synthesis and diffusion propagated along the array at a constant velocity of $v=0.62 \mathrm{~mm} \mathrm{~h}^{-1}$ (1.55 compartment $\mathrm{h}^{-1}$ with compartment unit length $L_{2}=0.4 \mathrm{~mm}$; Fig. 1d). The expression dynamics in the array exhibited sequential switching to a high steady-state level, with a delay of $\Delta t \approx 0.7 \mathrm{~h}$ between neighbouring compartments (Fig. 1c), and a gradual decrease in protein synthesis due to intrinsic decay in cell extract activity (Methods). In a moving frame of reference, $\xi=x-v t$, the spatiotemporal levels collapsed to a single step-function, $\operatorname{Tanh}((\xi / \eta))$, with $\eta=0.2 \mathrm{~mm}$ (half a compartment; Fig. 1e). The front velocity is consistent with the scaling $v \propto \sqrt{k D} \approx 0.34 \mathrm{~mm} \mathrm{~h}^{-1}$. Here, $k \approx 5.4 \mathrm{~h}^{-1}$ is the growth rate of the autocatalytic reaction estimated experimentally from the initial protein synthesis rate, 
a

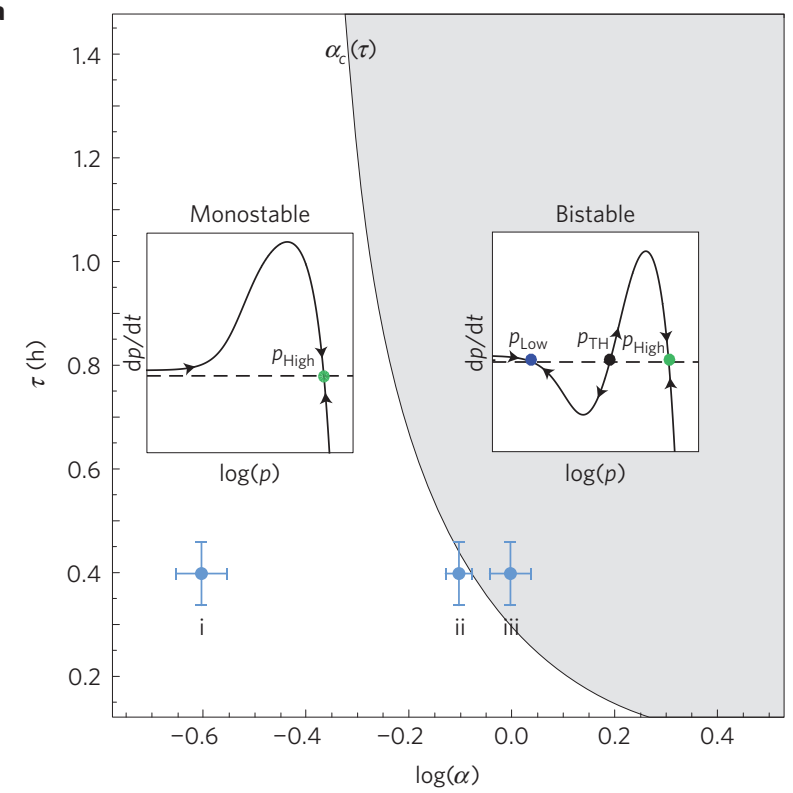

b

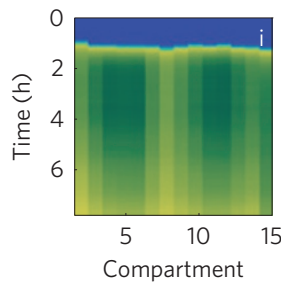

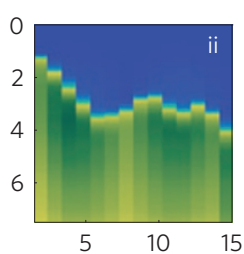

Compartment

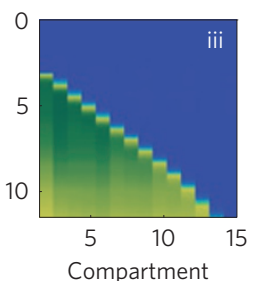

c

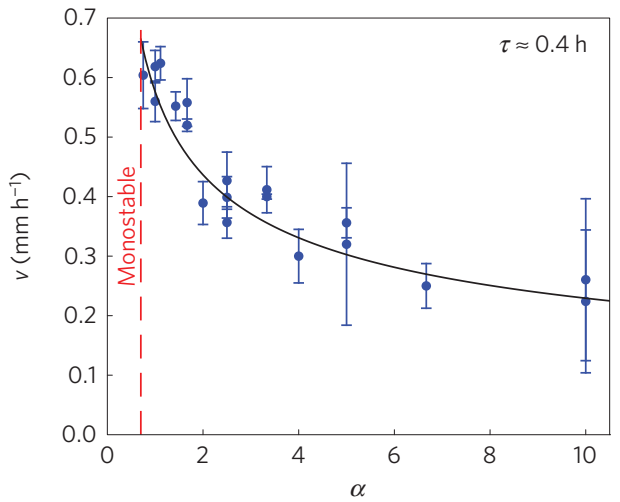

d
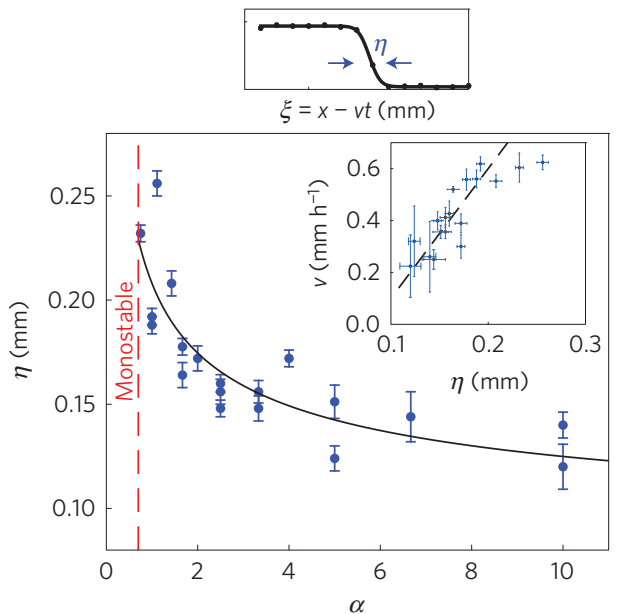

Figure 2 | Propagation dynamics regulated by gene circuit. a, A model-based phase diagram in parameter space of lifetime $\tau$ and gene ratio $\alpha=G_{1} / G_{A}$ showing a region of monostability with a single fixed point (left inset), and a region of bistability with two stable ( $p_{\text {High }}$ and $p_{\text {Low }}$ ) and one unstable ( $p_{\text {TH }}$ ) fixed points (right inset). Error bars are estimated from variation in lifetime and gene ratio (Supplementary Fig. 7). Points labelled i-iii are described in $\mathbf{b}$. b. Spacetime plots of propagator in the DNA array for the three points marked in a in the (i) monostable, (ii) close to transition and (iii) bistable regimes. Blue (green) colour represents low (high) protein level. c, Propagation velocity as a function of $\alpha$. Plot shows three different experiments. Solid line is a fit to $v=v_{0} \alpha^{-\beta}, \beta=0.4 \pm 0.07, v_{0}=0.59 \pm 0.02 \mathrm{~mm} \mathrm{~h}^{-1}$. Each data point is taken from a single experiment with front propagation over a number of compartments. The velocity is extracted by fitting a linear slope to the expression onset time in each compartment as a function of compartment location. The error bar is given by the velocity fit error for each experiment. d, Propagation front width $\eta$ as a function of $\alpha$, calculated from a fit of the collapsed and normalized propagating front. Solid line is a fit to $\eta=0.07\left(1+2 \alpha^{-0.4}\right)(\mathrm{mm})$. Inset: velocity as a function of front width; black line is a linear fit. Each data point is taken from a single experiment, and the error bar is given by the width fit error for each experiment.

$\partial_{t} p_{i}=k p_{i}$, which fits to an exponential growth with a rate $k$ at low protein concentrations (Supplementary Fig. 5). This scaling, also known as Luther's formula ${ }^{16}$, is common to autocatalytic reactiondiffusion systems in chemistry and biology, and has been observed over many orders of magnitude (Fig. 1f and Supplementary Table 1), including gene expression fronts propagating through the anteroposterior axis of developing vertebrates ${ }^{26}$, mitosis waves in Xenopus eggs ${ }^{11}$, cAMP waves spatial organization of amoeba ${ }^{27}$, calcium waves in the heart ${ }^{28}$, and action potentials in neurons ${ }^{16}$. The gene expression propagation velocity in the array is slower than in vitro enzymatic front waves ${ }^{10,14,20} v \approx 3 \mathrm{~mm} \mathrm{~h}^{-1}$, and chemical reactions such as Belousov-Zhabotinsky ${ }^{16} v \approx 140 \mathrm{~mm} \mathrm{~h}^{-1}$.

The theoretical model of the gene circuit, $f\left(p_{i}\right)$ (Supplementary Equation 40), revealed a transition from a bistable regime supporting front propagation to a monostable regime, which occurs along a saddle-node bifurcation line $\alpha_{c}(\tau)$ in the parameter space of protein lifetime, $\tau$, and gene ratio, $\alpha=\left(G_{\mathrm{I}} / G_{\mathrm{A}}\right)$, of inhibitor and activator (Fig. 2a and Supplementary Fig. 6). The inhibitor gene concentration was fixed in theory and experiment. A bistable regime requires a high inhibitor concentration to suppress the autocatalytic leak, which corresponds to a long protein lifetime, or high gene ratio. A monostable regime is found at a low gene ratio, or short lifetime. In the bistable regime, $\alpha>\alpha(\tau)$, the activator flow chart exhibits two stable fixed points, corresponding to high and low protein levels (Fig. 2a, right inset). There is an additional unstable fixed point, $p_{\mathrm{TH}}$, which is the concentration threshold for switching the compartment between the two expression states. In crossing the saddle-node bifurcation line, the two fixed points $p_{\mathrm{TH}}$ and $p_{\mathrm{Low}}$ are annihilated, and in the monostable regime, $\alpha<\alpha_{c}(\tau)$, there is a single fixed point of high levels $p_{\text {High }}$ (Fig. $2 \mathrm{a}$, left inset). Notably, the competition over gene expression machinery is not necessary to achieve bistability, but expands the bistable region in parameter space (Supplementary Fig. 6) ${ }^{29}$.

We fabricated multiple arrays with identical lifetime $\tau \approx 0.4 \mathrm{~h}$, and different gene ratios $0.1<\alpha<10$, with $5-10 \%$ variation in lifetime and gene ratio $^{13}$ (Supplementary Fig. 7). We observed two distinct regimes, as predicted in the phase diagram. At low inhibitor ratio, $\alpha<0.7$, expression initiated simultaneously in all the compartments and reached high levels without observable propagation, corresponding to the monostable regime (Fig. 2b(i)). For $0.7<\alpha<10$, we observed a travelling gene expression front (Fig. 2b(iii)). The compartments maintained low expression levels $\left(p_{\text {Low }}\right)$ until triggered by the propagating activator, corresponding to the bistable regime. Near the transition line, $\alpha \approx 0.7$, we observed signal propagation for a finite time, after which expression initiated simultaneously in the entire array (Fig. $2 \mathrm{~b}(\mathrm{ii})$ ). This suggests the 
a

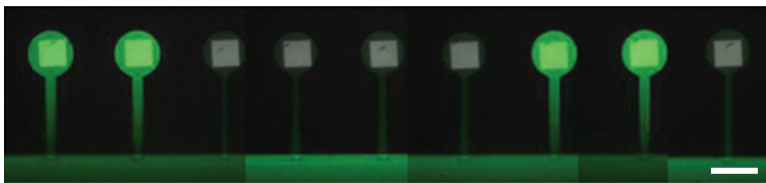

c

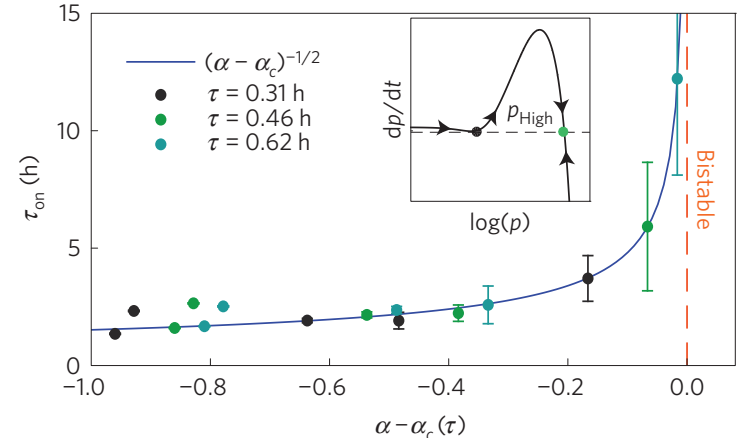

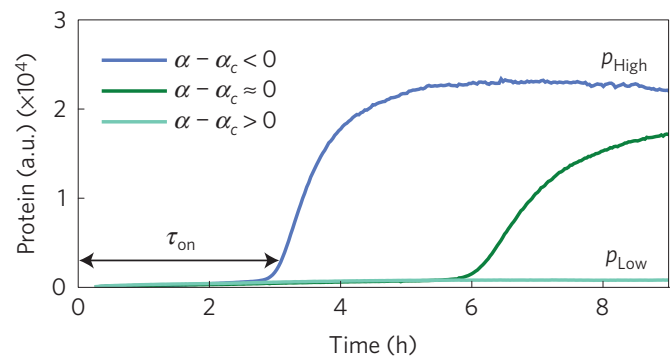

d

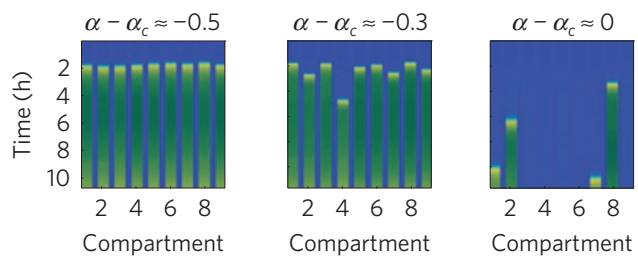

Figure 3 | Emergent fluctuations in single compartments near the transition. a, Overlay image of GFP and DNA in single compartments after 10 h of expression. The nine compartments were patterned with the bistable gene circuit at the same gene ratio $\alpha$. Scale bar, $100 \mu m$. $\mathbf{b}, \mathrm{Kinetics}$ of the circuit in single compartments with $\tau=1 \mathrm{~h}$ for three different gene ratios. $\mathbf{c}$, Reaction onset time in the compartments as a function of $\alpha-\alpha_{c}(\tau)$ for three different lifetimes. Each lifetime exhibited a different $\alpha_{c}$, and the function was subtracted accordingly (Supplementary Information). Errors are taken from nine measurements for each point. Standard deviation increased up to 35\% as the DNA ratio approached the transition to the bistable region $\alpha \rightarrow \alpha_{c}$. d, Spacetime plots showing nine repeats of single-compartment circuit kinetics, with $\tau=0.62 \mathrm{~h}$, for three different gene ratios as denoted. Blue (green) colour represents low (high) protein level.

system is in the monostable regime, but exhibits long onset times. Our model implies a third regime for $\alpha>10$, in which the system supports front propagation of $p_{\text {Low }}$ into $p_{\text {High }}$. This is not observable because the initial state of the array is $p_{\text {Low }}$ and there is a constant flux of activator proteins from the starter compartment (Supplementary Fig. 6).

We next explored the propagation velocity and front width as a function of gene ratio $\alpha$ in the bistable regime (Fig. 2c,d and Supplementary Fig. 8, $\alpha>0.7)$. At high inhibitor to activator gene ratio, $\alpha \gg \alpha_{c}$, the propagation was slow, $v(\alpha=10)=0.2 \mathrm{~mm} \mathrm{~h}^{-1}$. As we approached the bifurcation line, gene ratio in the compartment decreased, $\alpha \rightarrow \alpha_{c}$, positive feedback was triggered at lower activator concentration, and the velocity increased by a factor of three at $\alpha=0.75$. Our model shows the threshold concentration is low near the bifurcation point, $p_{\mathrm{TH}}\left(\alpha_{c}\right) \rightarrow p_{\text {Low }}$, hence compartments are easily excited to high activator levels, which is consistent with the observed faster propagation. The velocity is fitted with a power law, $v=v_{0} \alpha^{-\beta}$, with $\beta=0.4 \pm 0.07, v_{0}=0.59 \pm 0.02 \mathrm{~mm} \mathrm{~h}^{-1}$ (Fig. 2c), which is close to the classical Luther scaling, $v=(D k)^{\frac{1}{2}} \propto \alpha^{-\frac{1}{2}}$, where the autocatalytic growth rate is proportional to the activator gene concentration and inversely proportional to the gene ratio. The front width broadened with decreasing gene ratio $\alpha$, but remained sharper than a compartment, $0.125<\eta<0.25 \mathrm{~mm}$ (Fig. 2d). The front width exhibited a linear dependence on the front velocity $\eta=\eta_{0}+\left(v / k_{0}\right)$ (Fig. 2d), and $k_{0}=4.2 \mathrm{~h}^{-1}$ is close to the measured autocatalytic reaction rate, $5.4 \mathrm{~h}^{-1}$. This is consistent with the observed linear dependence, $\eta \propto v$, in other bistable models ${ }^{18}$.

We next investigated the behaviour of the gene circuit in isolated compartments for varying lifetime $\tau$ and gene ratio $\alpha$ (Fig. 3a). We observed a transition between a monostable regime, $\alpha<\alpha_{c}$, in which the compartments spontaneously switched to high activator levels, and a bistable regime, $\alpha>\alpha_{c}$, in which the compartments remained at low levels (Fig. $3 b$ ), consistent with the propagation behaviour. The expression onset time in the monostable regime diverged near the bifurcation $\alpha_{c}(\tau)$, where the inhibition is sufficiently strong to prevent the onset of the positive feedback, beyond the duration of the experiment $(\sim 13 \mathrm{~h}$; Fig. $3 \mathrm{~b}, \mathrm{c})$. The transition point $\alpha_{c}(\tau)$ decreased with increasing lifetime $\tau$, consistent with the phase diagram (Fig. 2a and Supplementary Figs 9 and 10).
The divergence was consistent with a universal power-law scaling, $\tau_{\text {on }} \sim\left(\alpha-\alpha_{c}(\tau)\right)^{-1 / 2}$, as expected near a saddle-node bifurcation ${ }^{30}$. Furthermore, we found a large variation in the expression onset time near the bifurcation (Fig. 3a,d and Supplementary Fig. 10). At $\alpha-\alpha_{c}=-0.5$ the expression onset time was $\tau_{\text {on }}=2 \mathrm{~h}$, with less than $5 \%$ variation between compartments. Near the transition, $\alpha \approx \alpha_{c}$, the variation was greater than $35 \%$, with only $40 \%$ of the compartments initiating expression in $13 \mathrm{~h}$ (Supplementary Fig. 11). The observed variation is surprising because the number of DNA copies in each compartment is large ${ }^{13}, \sim 10^{7}$, and therefore stochastic effects should be negligible. However, the proximity to a transition point with diverging onset times amplifies small differences in gene ratio between compartments. This mechanism can play a crucial role in biological processes, such as development, which relies on spontaneous symmetry breaking ${ }^{31}$. To summarize, we have demonstrated front propagation of gene expression along an array of artificial cells using programmable integrated gene circuits. The spatial organization of DNA circuits together with short interaction length, set by the array geometry, will allow integrating long-range signalling with local information processing reactions based on gene expression, in analogy to multicellular systems, electronic circuits and neuronal networks.

\section{Methods}

Methods and any associated references are available in the online version of the paper.

Received 7 April 2015; accepted 10 August 2015; published online 21 September 2015

\section{References}

1. Gregor, T., Fujimoto, K., Masaki, N. \& Sawai, S. The onset of collective behavior in social amoebae. Science 328, 1021-1025 (2010).

2. Bassler, B. L. \& Losick, R. Bacterially speaking. Cell 125, 237-246 (2006).

3. Hubaud, A. \& Pourquié, O. Signalling dynamics in vertebrate segmentation. Nature Rev. Mol. Cell Biol. 15, 709-721 (2014).

4. Kandel, E., Schwartz, J., Jessell, T. M., Seigelbaum, S. A. \& Hudspeth, A. J. Principles of Neural Science (McGraw-Hill Professional, 2012).

5. Bulter, T. et al. Design of artificial cell-cell communication using gene and metabolic networks. Proc. Natl Acad. Sci. USA 101, 2299-2304 (2004). 
6. Basu, S., Gerchman, Y., Collins, C. H., Arnold, F. H. \& Weiss, R. A synthetic multicellular system for programmed pattern formation. Nature $\mathbf{4 3 4}$, 1130-1134 (2005).

7. Matsuda, M., Koga, M., Nishida, E. \& Ebisuya, M. Synthetic signal propagation through direct cell-cell interaction. Sci. Signal. 5, ra31 (2012).

8. Danino, T., Mondragón-Palomino, O., Tsimring, L. \& Hasty, J. A synchronized quorum of genetic clocks. Nature 463, 326-330 (2010).

9. Isalan, M., Lemerle, C. \& Serrano, L. Engineering gene networks to emulate Drosophila embryonic pattern formation. PLoS Biol. 3, e64 (2005).

10. Loose, M., Fischer-Friedrich, E., Ries, J., Kruse, K. \& Schwille, P. Spatial regulators for bacterial cell division self-organize into surface waves in vitro. Science 320, 789-792 (2008).

11. Chang, J. B. \& Ferrell, J. E. Mitotic trigger waves and the spatial coordination of the Xenopus cell cycle. Nature 500, 603-607 (2013).

12. Keber, F. C. et al. Topology and dynamics of active nematic vesicles. Science 345, 1135-1139 (2014).

13. Karzbrun, E., Tayar, A. M., Noireaux, V. \& Bar-Ziv, R. H. Programmable on-chip DNA compartments as artificial cells. Science 345, 829-832 (2014).

14. Zadorin, A. S., Rondelez, Y., Galas, J.-C. \& Estevez-Torres, A. Synthesis of programmable reaction-diffusion fronts using DNA catalyzers. Phys. Rev. Lett. 114, 068301 (2015).

15. Cross, M. \& Hohenberg, P. Pattern formation outside of equilibrium. Rev. Mod. Phys. 65, 851-1112 (1993).

16. Tyson, J. J. \& Keener, J. P. Singular perturbation theory of traveling waves in excitable media (a review). Physica D 32, 327-361 (1988).

17. Fisher, R. A. The wave of advance of advantageous genes. Ann. Eugen. 7, 355-369 (1937)

18. Keener, J. P. \& Sneyd, J. Mathematical Physiology I (Springer, 1998).

19. Winfree, A. T. Spiral waves of chemical activity. Science 175, 634-636 (1972).

20. Bauer, G. J., McCaskill, J. S. \& Otten, H. Traveling waves of in vitro evolving RNA. Proc. Natl Acad. Sci. USA 86, 7937-7941 (1989).

21. Noireaux, V., Bar-Ziv, R. \& Libchaber, A. Principles of cell-free genetic circuit assembly. Proc. Natl Acad. Sci. USA 100, 12672-12677 (2003).

22. Noireaux, V. \& Libchaber, A. A vesicle bioreactor as a step toward an artificial cell assembly. Proc. Natl Acad. Sci. USA 101, 17669-17674 (2004).

23. Weitz, M. et al. Diversity in the dynamical behaviour of a compartmentalized programmable biochemical oscillator. Nature Chem. 6, 295-302 (2014).
24. Niederholtmeyer, H., Stepanova, V. \& Maerkl, S. J. Implementation of cell-free biological networks at steady state. Proc. Natl Acad. Sci. USA 110, 15985-15990 (2013)

25. Kim, J., White, K. S. \& Winfree, E. Construction of an in vitro bistable circuit from synthetic transcriptional switches. Mol. Syst. Biol. 2, 68 (2006).

26. Bajard, L. et al. Wnt-regulated dynamics of positional information in zebrafish somitogenesis. Development 141, 1381-1391 (2014).

27. Kessler, D. \& Levine, H. Pattern formation in dictyostelium via the dynamics of cooperative biological entities. Phys. Rev. E 48, 4801-4804 (1993).

28. Meyer, T. Cell signalling by second messenger waves. Cell 64, 675-678 (1991).

29. Rondelez, Y. Competition for catalytic resources alters biological network dynamics. Phys. Rev. Lett. 108, 018102 (2012)

30. Strogatz, S. H. International Edition Nonlinear Dynamics and Chaos: With Applications to Physics, Biology, Chemestry and Engeneering (Westview Press, 2001).

31. Artavanis-Tsakonas, S., Rand, M. D. \& Lake, R. J. Notch signaling: Cell fate control and signal integration in development. Science 284, 770-776 (1999).

\section{Acknowledgements}

We thank S. S. Daube for helpful discussions. V.N. thanks J. Garamella, R. Marshall and M. Rustad for technical help. This work was supported by: the Israel Science Foundation, the Minerva Foundation, and the Volkswagen Foundation (R.H.B.-Z.); the US-Israel Binational Science Foundation (R.H.B.-Z. and V.N.).

\section{Author contributions}

All authors contributed to all aspects of this work.

\section{Additional information}

Supplementary information is available in the online version of the paper. Reprints and permissions information is available online at www.nature.com/reprints.

Correspondence and requests for materials should be addressed to R.H.B.-Z.

\section{Competing financial interests}

The authors declare no competing financial interests. 


\section{Methods}

Biochip fabrication. The fabrication and assembly protocol of the DNA compartments is similar to previous work ${ }^{13}$. Each array was composed of 15 interconnected circular compartments etched 2.5-3 $\mu \mathrm{m}$ deep into a silicon wafer and connected to a main flow channel (Supplementary Fig. 1A). Silicon wafers $\left(5^{\prime \prime}, 0.525 \mathrm{~mm}\right.$ thickness, test grade, $\langle 100\rangle$, p-type, University Wafers) were used as substrates. The compartments had a radius of $50 \mu \mathrm{m}$ and were connected through a capillary channel, $10-15 \mu \mathrm{m}$ in width to each other and to a perpendicular flow channel, $50 \mu \mathrm{m}$ deep and $900 \mu \mathrm{m}$ wide (Fig. 1a and Supplementary Fig. 1B). The flow channel had an inlet at one side and a $50-\mu \mathrm{m}$-wide serpentine ending with an outlet at the other side. The silicon wafer was patterned using standard lithography techniques (Photoresists S1818, AZ4562 MicroChem) and etched using reactive ion etching in an ICP-RIE (Surface Technology Systems) using the Bosch process ${ }^{32}$ for deep features $(>5 \mu \mathrm{m})$. The compartment and flow channel were etched in two separate steps. The inlets and outlets of each device were drilled using a drill machine (Proxxon, TBM 220). The device was cleaned using a base piranha solution followed by short incubation in hydrofluoric acid. The device was coated with a $\sim 50 \mathrm{~nm} \mathrm{SiO}_{2}$ layer deposited by low-temperature ALD (atomic layer deposition, FIJI F200, Cambridge Nanotech).

Biocompatible photoactivable monolayer assembly. The $\mathrm{SiO}_{2}$-coated device was incubated with a biocompatible photoactivable polymer solution that self-assembled into a monolayer on the $\mathrm{SiO}_{2}$ surface, for subsequent activation by ultraviolet light and binding of biomolecules ${ }^{33}$. The polymer is composed of a polyethylene glycol backbone with a Nvoc-protected amine at one end, and a trialkoxysilane function at the other end ${ }^{33}$. Incubation was carried for $20 \mathrm{~min}$ at $0.2 \mathrm{mg} \mathrm{ml}^{-1}$ in toluene, followed by rinsing in toluene and drying.

Lithography patterning for DNA assembly. The device was mounted on the translation stage of an inverted microscope (Zeiss Axiovert 200). Ultraviolet light from a fluorescent light source (EXFO X-Cite 120Q) was illuminated through a rectangular pinhole and a $365 \mathrm{~nm}$ band-pass filter (Chroma), and focused on the substrate using a $\times 60$ objective. The exposure time was set to yield a total $2.5 \mathrm{~J} \mathrm{~cm}^{-2}$. Reactive amine groups on the biocompatible photoactivable monolayer were exposed in surface patterns illuminated by ultraviolet light ${ }^{33}$. Biotin $N$-hydroxysuccinimidyl ester (biotin-NHS) dissolved in a borate buffered saline $\left(0.5 \mathrm{mg} \mathrm{ml}^{-1}\right)$ was incubated on the chip for $15 \mathrm{~min}$. The biotin-NHS covalently bound to the exposed amine groups on the exposed monolayer ${ }^{33}$. We thus attained a surface patterned with biotin.

DNA deposition and brush assembly. Linear DNA fragments were produced by polymerase chain reaction (PCR) with KAPA HiFi HotStart ReadyMix (KK2601, KAPA BIOSYSTEMS), using one primer with biotin and another with Alexa Fluor 647 , both attached at the $5^{\prime}$-end (IDT). The biotin primer was located downstream of the transcription terminator. PCR products were cleaned using Promega Wizard SV-Gel and PCR Clean-Up. DNA was conjugated to streptavidin (SA) by mixing in solution in a molar ratio of 1:1.5 DNA:SA. The final DNA solution contained SA-conjugated DNA at a concentration of 100-300 nM in phosphate buffered saline (PBS).

Nanolitre DNA-SA droplets were individually deposited onto the reactor chambers using the GIX Microplotter II (Sonoplot) and $60-\mu \mathrm{m}$-diameter micropipettes. The DNA-SA solutions were incubated on the device for $1-2 \mathrm{~h}$ in PBS. During incubation the DNA formed a dense brush on the surface. The brush density was of the order of $10^{3} \mathrm{DNA} \mu \mathrm{m}^{-2}$ (refs 33,34). The promoter orientation of the DNA was towards the surface of the DNA brush ${ }^{35}$. The gene size varied in the range $700-1,500 \mathrm{bp}$. The distance between the DNA top and the promoter is about 100-200 bp and a similar distance between the terminator and the DNA end attached to the surface. Finally, DNA brushes were localized to the lithographically patterned areas inside the etched compartments (Fig. 1a and Supplementary Fig. 1). The device was then bathed in PBS, and then in water to remove excess adsorbed DNA. The device was then gently dried with nitrogen.

All E. coli cell-free transcription-translation reaction (CFE). In this study we used a CFE that is a cytoplasmic extract from E. coli strain BL21 Rosetta2 (Novagen) according to a procedure described previously ${ }^{36}$. The cell-free reactions were composed of $33 \%$ (volume) crude extract and $66 \%$ (volume) of water, DNA and buffer with the following final composition: $50 \mathrm{mM}$ HEPES pH 8, $1.5 \mathrm{mM}$ ATP $1.5 \mathrm{mM}$ GTP, $0.9 \mathrm{mM}$ CTP, $0.9 \mathrm{mM}$ UTP, $0.2 \mathrm{mg} \mathrm{ml}^{-1}$ tRNA, $0.26 \mathrm{mM}$ coenzyme A, $0.33 \mathrm{mM}$ NAD, $0.75 \mathrm{mM}$ cAMP, $0.068 \mathrm{mM}$ folinic acid, $1 \mathrm{mM}$ spermidine, $30 \mathrm{mM}$ 3-phosphoglyceric acid, $2 \mathrm{mM}$ DTT, $1.5 \mathrm{mM}$ amino acids, $6.5 \mathrm{mM} \mathrm{Mg}$-glutamate, $100 \mathrm{mM}$ K-glutamate and 2\% PEG 8000. During extract preparation, the endogenous DNA and mRNA were degraded. The cell extract provided the transcription and the translation machineries necessary for gene expression. Transcription was driven by the endogenous E. coli RNA polymerase and thus allowed us to use the entire repertoire of the E. coli regulation toolbox ${ }^{37,38}$. The CFE contained active proteases and ribonucleases. Previously, we studied the stability of proteins and mRNA in our cell-free system ${ }^{13,36,39}$. Proteins without a degradation tag were stable with no observed degradation. The protein GamS, was added to all of the reactions at a concentration of $3 \mu \mathrm{M}$ to minimize the degradation of linear DNA by the $3^{\prime}$ exonuclease activity of the RecBCD complex ${ }^{40}$ which was present in the CFE. The CFE was supplemented with 3-phosphoglyceric acid (3-PGA) for ATP regeneration ${ }^{36}$. The 3-PGA is a natural substrate to $E$. coli and therefore no enzyme was added to the extract.

Sealing the device and flow of CFE. The carved side of the device was sealed with a PDMS-coated glass coverslip and magnets. Magnets embedded in a punched PDMS were attached to the backside of the device (the untreated side of the device), aligned to the drilled inlet and outlet. The device inlet was connected using elastic tubing to a reservoir of PBS cooled to $2-4{ }^{\circ} \mathrm{C}$ with a cooling circulator (Huber ministat). The outlet was connected to a syringe pump (Harvard Apparatus Pico Plus). The device was placed on a microscope, in an incubating chamber $\left(30^{\circ} \mathrm{C}\right)$. Once the syringe pump was turned on, vacuum was applied and PBS flowed into the main flow channel and entered by capillarity into the compartments within a minute, pushing out air through the PDMS. The experiment began by exchanging PBS with CFE flowing in the main channel at a rate of $\sim 0.2 \mu \mathrm{lmin}^{-1}$ and diffusing through the thin capillaries into DNA compartments. Constant flow was maintained during the experiment, which was carried on for $12-15 \mathrm{~h}$. During this long period there is a slow loss of extract activity, which is observed in a decaying expression envelope for all of the compartments.

Imaging. Cell-free expressed GFP in the compartments was imaged on an inverted microscope (Zeiss Axiovert 200) equipped with an automated translation stage, a sensitive wide view camera (ANDOR Neo sCMOS, Andor Technology) through a $\times 10$ objective (Zeiss), and fluorescent excitation (Hg-illumination lamp (X-Cite 120) through a GFP filter set $(488 \mathrm{nM})$.

Gene circuit design and assembly. DNA constructs used in this work are described in Supplementary Table 2. Their assembly into single gene constructs and gene networks is described in Supplementary Table 3, as well as in Supplementary Fig. 3. All the plasmids were constructed from the pBEST-Luc plasmid (Promega), with the UTR1 (untranslated region). DNA constructs (promoter gene) were assembled in vitro by PCR using a primer coding for the promoter and a plasmid template coding for the gene. This was followed by a second PCR with biotinylated primers and primers with Alexa Fluor 647, as previously described.

The bistable gene circuit (Fig. 1a inset, Supplementary Fig. 3 and Supplementary Tables 2-3) was constructed using an autocatalytic element and its inhibitor ${ }^{25,41-44}$ :

Activator (P): $\mathrm{P}_{28}-\sigma^{28}$, autocatalytic construct expressing the sigma-28 transcription factor ${ }^{37}$ under its own promoter, $\mathrm{P}_{28}$, thereby forming a positive feedback loop.

Inhibitor (I): $\mathrm{P}_{70}-\mathrm{A} \sigma^{28}$, constitutively expressing the anti-sigma factor 28 (FlgM; ref. 45) under a $\sigma^{70}$ promoter ${ }^{37}$. The anti-sigma protein binds the sigma transcription factor with high affinity and forms a complex $(\mathbf{C})$, and thus sequesters the binding of sigma-28 to its prompter, inhibiting the positive feedback.

In the experiment the gene ratio $\alpha=\left(G_{I} / G_{\mathrm{p}}\right)$ of inhibitor $G_{\mathrm{I}}$ and autocatalytic $G_{\mathrm{p}}$ elements of the circuit were varied (Supplementary Fig. 3). The inhibitor and reporter constructs were kept at a constant concentration while the activator concentration was changed. To maintain the total DNA of each brush constant we used a non-coding 'dummy' DNA of the same length and concentration as the activator construct.

\section{References}

32. Wang, X., Zeng, W., Lu, G., Russo, O. L. \& Eisenbraun, E. High aspect ratio Bosch etching of sub- $0.25 \mu \mathrm{m}$ trenches for hyperintegration applications. J. Vac. Sci. Technol. B 25, 1376-1381 (2007).

33. Buxboim, A. et al. A single-step photolithographic interface for cell-free gene expression and active biochips. Small 3, 500-510 (2007).

34. Bracha, D., Karzbrun, E., Shemer, G., Pincus, P. A. \& Bar-Ziv, R. H. Entropy-driven collective interactions in DNA brushes on a biochip. Proc. Natl Acad. Sci. USA 110, 4534-4538 (2013).

35. Daube, S. S., Bracha, D., Buxboim, A. \& Bar-Ziv, R. H. Compartmentalization by directional gene expression. Proc. Natl Acad. Sci. USA 107, 2836-2841 (2010).

36. Shin, J. \& Noireaux, V. Efficient cell-free expression with the endogenous E. coli RNA polymerase and sigma factor 70. J. Biol. Eng. 4, 8 (2010).

37. Shin, J. \& Noireaux, V. An E. coli cell-free expression toolbox: Application to synthetic gene circuits and artificial cells. ACS Synth. Biol. 1, 29-41 (2012).

38. Caschera, F. \& Noireaux, V. Synthesis of $2.3 \mathrm{mg} / \mathrm{ml}$ of protein with an all Escherichia coli cell-free transcription-translation system. Biochimie 99, 162-168 (2014). 
39. Karzbrun, E., Shin, J., Bar-Ziv, R. H. \& Noireaux, V. Coarse-grained dynamics of protein synthesis in a cell-free system. Phys. Rev. Lett. 106, 048104 (2011).

40. Karu, A. E., Sakaki, Y., Echols, H. \& Linn, S. The $\gamma$ protein specified by bacteriophage $\gamma$. Structure and inhibitory activity for the recBC enzyme of Escherichia coli. J. Biol. Chem. 250, 7377-7387 (1975).

41. Ferrell, J. E. Tripping the switch fantastic: How a protein kinase cascade can convert graded inputs into switch-like outputs. Trends Biochem. Sci. 21, 460-466 (1996)
42. Gardner, T. S., Cantor, C. R. \& Collins, J. J. Construction of a genetic toggle switch in Escherichia coli. Nature 403, 339-342 (2000).

43. Chang, D.-E. et al. Building biological memory by linking positive feedback loops. Proc. Natl Acad. Sci. USA 107, 175-180 (2010).

44. Huang, D., Holtz, W. J. \& Maharbiz, M. M. A genetic bistable switch utilizing nonlinear protein degradation. J. Biol. Eng. 6, 9 (2012).

45. Hughes, K. T. \& Mathee, K. The anti-sigma factors. Annu. Rev. Microbiol. 52, 231-286 (1998) 